\title{
A Single Nucleotide Polymorphism of Follicle-Stimulating Hormone Receptor Gene in Turkish Women With Polycystic Ovary Syndrome: A Cross-Sectional Study
}

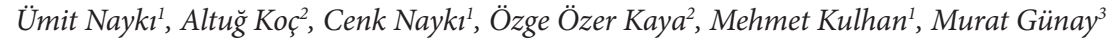

${ }^{1}$ Erzincan University, School of Medicine, Department of Obstetrics and Gynecology, Erzincan, Turkey ${ }^{2}$ Tepecik Training and Research Hospital, Department of Genetic Research, İzmir, Turkey ${ }^{3}$ Erzincan University, School of Medicine, Department of Biochemistry, Erzincan, Turkey

Corresponding author \& address: ${ }^{1}$ Erzincan University, School of Medicine, Department of Obstetrics and Gynecology, Erzincan, Turkey e-mail:drumit75@hotmail.com
Received: 04 Jan 2017

Accepted: 28 June 2017

DOI: 10.5799 /jcei. 333378

\section{A B S T R A C T}

Objective: The aim of this study was to evaluate both the frequency of Asn680Ser polymorphism of follicle stimulating hormone receptor (FSHR) and whether this polymorphism is associated with polycystic ovary syndrome (PCOS) in a Turkish population.

Patients and Methods: 100 patients with PCOS and 100 controls were recruited from Eastern Turkey. Venous blood samples were obtained for genetic study, hormone profile, glucose and insulin assays. Asn680Ser polymorphism of FSHR was analyzed by polymerase chain reaction (PCR) amplification and direct DNA sequencing. Two groups were compared for body mass index (BMI), modified Ferriman-Gallwey ( $\mathrm{mFG}$ ) score, fasting glucose, fasting insulin, insulin resistance, hormone and lipid status. Then, all patients were divided into subgroups for genotypes of FSHR polymorphism. These subgroups were also compared for these mentioned parameters.

Results: The difference between women with PCOS and controls in allelic frequencies for Asn680Ser polymorphism of FSHR were not significant. When this single nucleotide polymorphism (SNP) at codon 680 was separately analyzed, no significant difference was found in the genotype frequency among the Asn680Asn, Asn680Ser, andSer680Ser subgroups. There were no significant differences in BMI, mFG score, levels of fasting glucose and fasting insulin, HOMA-IR, and also levels of FSH, luteinizing hormone, estradiol, prolactin, thyroid stimulating hormone, total testosterone among the PCOS patients carrying different genotypes of Asn680Ser polymorphism

Conclusion: there is no association between Asn680Ser polymorphism of FSHR and PCOS in Turkish women from eastern provinces of Turkey. In addition, this SNP seems not to be associated with BMI, IR and PCOS-associated endocrine hormones.

Keywords: Polycystic ovary syndrome, follicle stimulating hormone receptor, single nucleotide polymorhism

\section{INTRODUCTION}

Polycystic ovary syndrome (PCOS), is a complex endocrine disorder, characterized by hyperandrogenism, anovulation and polycystic ovary morphology [1]. The prevelance of the disease is estimated to be $5-10 \%$ in women of reproductive age [2]. Also, it has been established that there are ethnic and racial variations in the prevalence of PCOS and its symptoms [3]. It is the leading cause of anovulatory infertility and hirsutismus [4]. In addition, women with PCOS are susceptible to obesity, insulin resistance, hypertension, and dyslipidemia that lead to cardivascular diseases [5]. The pathogenesis of PCOS still remains unclear. However, environment and genetic background are considered to be the potential causative factors for the disease. Twin and familial studies revealed that PCOS is closely associated with genetic factors (6). Recently, it has been shown that there are multiple susceptibility genes encoding sex hormones or regulators of their activities, associated with $\operatorname{PCOS}[7,8]$.

Follicle-stimulating hormone (FSH), a secretion of pituitary gland, plays a crucial role in the follicle development, oocyte maturation and steroidogenesis regulation [9]. The action 
of FSH is mediated by FSH receptor (FSHR), a member of the G-protein coupled receptor family, which is only expressed by the granulosa cells of the ovary and Sertoli cells of the testis. The gene encoding for this receptor is located at chromosome $2 \mathrm{p} 21$ and consists of 10 exons, 9 introns [10]. Among many polymorphisms, found in the screening studies for mutations of the FSHR gene, the single nucleotide polymorphism (SNP) at position 680, occupied either by asparagine (ASN, N) or Serine (Ser, S) is of particular interest [11]. Since FSHR has an important role in signaling transmission of FSH, FSHR gene may be responsible for causing PCOS. On the basis of this approach, associations between FSHR gene polymorphisms and PCOS have been investigated. However, contradictory results are present in the literature. Gu et al revealed that the Asn680 Ser of FSHR was associated with PCOS in Korean women [9]. On the otherhand, Fu et al reported that the Asn680Ser polymorphism of FSHR was not a causative factor of PCOS in Chinese women [12]. All of these studies were conducted on different ethnic groups from different geographic regions. Moreover, some previous studies have showed the association of altered ovarian response with FSHR gene polymorphism at codon 680 and have suggested that this FSHR gene polymorhism can be used as a potential marker to predict poor ovarian response $[13,14]$. However, the reports from different populations such as the Netherlands and the UK have indicated no association between this polymorphism and poor ovarian response $[15,16]$. Thus, geography and race may be responsible for this discrepancyin the findings about the relation between FSHR-PCOS. In this cross-sectinal study, the frequency distribution of FSHR polymorphism at codon 680 was examined in a female population from the eastern provinces of Turkey and we also evaluated the association between this SNP and PCOS.

\section{PATIENTS AND METHODS}

\section{Subjects and clinical, hormonal and biochemical assessments}

100 patients with PCOS and 100 controls were recruited from Erzincan University, Faculty of Medicine, Department of Obstetrics and Gynecology from January 2014 to May 2015. All subjects and the controls were from Eastern Turkey and were age- matched. PCOS was defined on the basis of the Rotterdam criteria [17]: 1. Oligo-ovulation and/or anovulation 2. Clinical and/or biochemical signs of hyperandrogenism, and 3.Polycystic morphology of the ovaries. Oligomenorrhea was defined as irregular menstruel cycles that are longer than 35 days, and amenorrhea was defined as absence of menstruation for more than 3 months without pregnancy. Clinical hirsutismus was assessed using modified Ferriman-Gallwey score (mFG score) and defined to be present at a score of at least 8 [18]. Polycystic morphology of the ovaries was assessed by vaginal ultrasonography (Mindray DC-7T ultrasound machine, Shenzen-Mindray Bio-Medical Electronics Co.Ltd., China) and defined as the presence of 12 or more follicles in one or both ovaries, and/or increased ovarian volume of $>10$ $\mathrm{ml}$. All of the clinical measurements were performed by the same physician. Women who met at least two of these criteria were diagnosed as PCOS. Hyperprolactinemia, thyroid dysfunction, diabetes mellitus, Cushing syndrome, congenital adrenal hyperplasia, androgen secreting neoplasms were exclusion criteria. The control group included healthy fertile women with regular menstruel cycles who had no endocrine disorder related to PCOS. They had no clinical or biochemical signs of hyperandrogenism and polycystic ovaries. None of the subjects had been taking any medications that might affect hormone, lipid or carbonhydrate metabolism for at least 3 months prior to starting the study. The study was approved by the Ethics Committee of the Erzincan University and written informed consent was obtained from each subject.

The general medical data including menstruel history, fertility, age, height, weight, and findings of the clinical examinations were recorded. Body mass index (BMI) was calculated as weight/ height $^{2}\left(\mathrm{~kg} / \mathrm{m}^{2}\right)$ [19].

After a 12-hour overnight fast, venous blood samples were obtained for genetic study and for hormonal profile, glucose and insulin assays between 8-10 AM on 3th to the 5th day of menstruel cycles. For amenorrheic patients, blood samples were obtained after a progesterone-induced menstruation. Follicle stimulating hormone [FSH, reference value (RV): $2.5-10.2 \mathrm{mIU} / \mathrm{mL}$ ], luteinizing hormone (LH, RV: 1.9-12.5 mIU/mL), estradiol (E2, RV:19.5$144.2 \mathrm{mIU} / \mathrm{mL}$ ), total testosterone (TT, RV: $14-76 \mathrm{ng} / \mathrm{dL}$ ), thyroid stimulating hormone (TSH, RV: $0.55-4.78 \mu \mathrm{U} / \mathrm{mL}$ ), prolactine (PRL, RV:2.8-29.2 ng/mL) were measured by Advia Centaur Xp Immunoassay System (Siemens, Gemany). TT values over the cut-off level of $76 \mathrm{ng} / \mathrm{dl}$ were defined as biochemical hyperandrogenism.

Plasma levels of fasting glucose (RV: 74-100 mg/dL), total cholesterole (CHO, RV: 0-200 mg/dL), triglycerides (TG; RV: 0-150 mg/dL), high-density lipoprotein (HDL, RV: 40-60 mg/ $\mathrm{dL}$ ), low-density lipoprotein (LDL; RV:0-130 mg/dL) levels were all detected by AU2700 Chemistry Analyzer (Beckman Coulter, USA). Fasting insulin (RV: $0-17 \mu \mathrm{U} / \mathrm{mL}$ ), was measured by Immulite 2000 XPI Immunoassay System (Siemens, Germany). Insulin resistance (IR) was identified by the HOMA-IR index obtained from the following calculation: HOMA-IR =fasting insulin ( $\mathrm{uIU} / \mathrm{ml}) x$ fasting glucose $(\mathrm{mg} / \mathrm{dl}) / 405$, and the value of 2.5 was accepted to be the cut off point [20].

Each of the two groups (PCOS and control) were divided into three subgroups according to the genotypes of FSHR polymorphism: Asn-Asn (AA) as group 1, Asn-Ser(AS) as group 2 , and Ser-Ser(SS) as group 3. All of the above mentioned parameters were compared between the PCOS and control group and also, among subgroups.

\section{Genotyping}

Genomic DNA was extracted from peripheral whole blood using MagPurix Blood DNA Extraction Kit by MagPurix 12 (Zinexts Life Science Corp., Taiwan). The genotyping procedure consisted of polymerase chain reaction (PCR) amplification and 
SNP detection of the c.2039G>A (p.Ser680Asn) mutation (rs6166) of FSHR gene using following pair of primers:

\section{Forward}

5'TGTAAAACGACGGCCAGTGAGCAAGTGTGGCTGCTATG-3' and

Reverse

5'-CAGGAAACAGCTATGACCTGTAGAAGCACTGTCAGCTC-3' by direct sequencing.

PCR amplification was carried out in a volume of $25 \mu \mathrm{l}$ containing $100 \mathrm{ng}$ sample DNA; HelixAmpTM Ready-2x-MultiPlex (Ver. 2.0) master mix was used; $25 \mathrm{nM}$ of each primer; and $1 \mathrm{U}$ of Taq polymerase were added to master mix (Nanohelix, South Korea). The amplification protocol conditions selected were as follows: Initial denaturation at $95^{\circ} \mathrm{C}$ for $10 \mathrm{~min}$, followed by 35 cycles of denaturation at $95^{\circ} \mathrm{C}$ for $30 \mathrm{sec}$, annealing at $60^{\circ} \mathrm{C}$ for $45 \mathrm{sec}$, extension at $72{ }^{\circ} \mathrm{C}$ for $45 \mathrm{sec}$ and a final extension at 72 ${ }^{\circ} \mathrm{C}$ for $7 \mathrm{~min}$. PCR products were purified as follows: $5 \mu \mathrm{l}$ PCR products were treated with $2 \mu \mathrm{l}$ of ExoSAP-IT enzyme (USB Affymetrix, USA) at $37^{\circ} \mathrm{C}$ for $30 \mathrm{~min}$ and at $80^{\circ} \mathrm{C}$ for $15 \mathrm{~min}$. Sequence PCR (cycle sequencing) was done by reverse PCR primer (5 pmol) and BigDye Terminator v3.1 Cycle Sequencing Kit (Lifetechnologies, USA). The PCR conditions were as follows: At $96{ }^{\circ} \mathrm{C}$ for $1 \mathrm{~min}$, at $96^{\circ} \mathrm{C} 10 \mathrm{sec}$, at $50^{\circ} \mathrm{C}$ for $5 \mathrm{sec}$, at $60^{\circ} \mathrm{C}$ for $4 \mathrm{~min}$ the cycle was repeated 25 times. Products of sequence PCR was purified (second purification) by spin colon (ZR DNA Sequencing Clean-up Kit ${ }^{\mathrm{m}}$, Zymo Research, USA). Sanger sequencing was performed by capillary electrophoresis after $5 \mathrm{~min}$ denaturation (3500 Genetic Analyzer, Lifetechnologies, USA). The obtained sequences were analyzed using by SeqScape ${ }^{\oplus}$ Software v3.0.

\section{Statistical Analysis}

The statistical analysis was performed using the Statistical Package for the Social Sciences version 22 (SPSS Inc., Chicago, IL, USA). Student $t$-test or Mann-Whithey U test was used to analyze the differences of continuous variables between the two groups. ANOVA and Kruskal-Wallis tests were used to analyze the differences among subgroups. Genotype and allele frequencies for the PCOS and control groups were compared using the $X^{2}$ test conformed to Hardy-Weinberg equilibrium. Results were expressed as percentage and/or mean \pm standard deviation (SD). Statistical significance was set at $P<0.05$.

\section{RESULTS}

Some basic characteristics of the subjects were shown in Table 1. mFG score, plasma levels of LH, TT and fasting insulin, HOMA-IR were significantly higher in PCOS group than the controls $(P<0.001, P<0.001, P<0.001, P=0.001$, and $P<0.004$, respectively). There were no significant differences between the PCOS group and the controls in age, FSH, E2, PRL, TSH, fasting glucose, and lipid profile.

The difference between women with PCOS and controls in allelic frequencies for the polymorphism at codon Asn680 were not significant $(P=0,777 ; 0 \mathrm{R}=0,923)$. When the $\mathrm{SNP}$ at amino acid positions 680 was separately analyzed, the genotype paterns were distributed in PCOS patients versus controls as follows: $30 \%$ vs $32 \%$ for AA, $42 \%$ vs 34 for AS, $28 \%$ vs $34 \%$ for SS. No significant difference was found in genotype patterns between groups $\left(\chi^{2}=0.744, P=0.689\right)$ (Table 2$)$.

Table 1. Baseline characteristics of the subjects

\begin{tabular}{|c|c|c|c|}
\hline Variable & $\begin{array}{l}\text { Controls } \\
(n=100)\end{array}$ & $\begin{array}{c}\text { Cases } \\
(n=100)\end{array}$ & $\begin{array}{c}P \\
\text { value }\end{array}$ \\
\hline Age (year) & $30.94 \pm 6.88$ & $30.00 \pm 3.49$ & $0.392^{\mathrm{a}}$ \\
\hline BMI $\left(\mathrm{kg} / \mathrm{m}^{2}\right)$ & $23.13 \pm 2.55$ & $24.27 \pm 4.60$ & $0.130^{\mathrm{a}}$ \\
\hline mFG score & $0.66 \pm 1.64$ & $10.40 \pm 3.37$ & $0.000^{b}$ \\
\hline $\mathrm{FSH}(\mathrm{mIU} / \mathrm{mL})$ & $6.83 \pm 4.22$ & $5.25 \pm 1.56$ & $0.14^{b}$ \\
\hline $\mathrm{LH}(\mathrm{mIU} / \mathrm{mL})$ & $5.47 \pm 3.77$ & $10.70 \pm 6.15$ & $0.000^{b}$ \\
\hline $\mathrm{E} 2(\mathrm{mlU} / \mathrm{mL})$ & $44.98 \pm 30.57$ & $48.04 \pm 24.99$ & $0.263^{b}$ \\
\hline PRL (ng/mL) & $12.29 \pm 5.55$ & $14.32 \pm 6.76$ & $0.176^{\mathrm{b}}$ \\
\hline $\mathrm{TSH}(\mu \mathrm{U} / \mathrm{mL})$ & $1.99 \pm 1.07$ & $1.62 \pm 0.80$ & $0.075^{b}$ \\
\hline $\mathrm{TT}$ (ng/dL) & $40.93 \pm 21.31$ & $65.20 \pm 24.11$ & $0.000^{\mathrm{a}}$ \\
\hline Fasting glucose (mg/dL) & $88.34 \pm 11.14$ & $87.68 \pm 10.1$ & $0.671^{\mathrm{b}}$ \\
\hline Fasting insulin $(\mu \mathrm{U} / \mathrm{mL})$ & $7.79 \pm 3.93$ & $12.86 \pm 8.9$ & $0.001^{b}$ \\
\hline HOMA-IR & $1.62 \pm 0.72$ & $2.74 \pm 2.08$ & $0.004^{b}$ \\
\hline $\mathrm{CHO}(\mathrm{mg} / \mathrm{dL})$ & $171.3 \pm 41.5$ & $172.5 \pm 41.1$ & $0.892^{\mathrm{a}}$ \\
\hline TG (mg/dL) & $114.7 \pm 60.7$ & $104.1 \pm 52.0$ & $0.357^{b}$ \\
\hline $\mathrm{HDL}(\mathrm{mg} / \mathrm{dL})$ & $49.5 \pm 13.3$ & $52.3 \pm 14.3$ & $0.307^{\mathrm{b}}$ \\
\hline LDL (mg/dL) & $102.9 \pm 28.7$ & $103.7 \pm 29.5$ & $0.912^{b}$ \\
\hline
\end{tabular}

Data are presented as Mean \pm Standard deviation (SD)

BMI:Body mass index, mFG score: Modified Ferriman-Gallwey score, FSH:Follicle stimulating hormone, LH:Luteinizing hormone, E2:Estradiol, PRL:Prolactine, TSH:Thyroid stimulating hormone, TT: Total testosterone, HOMA-IR: Homoeostasis model assessment, CHO:Cholesterol, TG:Triglycerides, HDL: High-density lipoprotein, LDL:Low-density lipoprotein

aStudent's $t$ test

${ }^{\mathrm{b}}$ Mann-Whitney $\mathrm{U}$ test

Table 2. Genotype distribution and allele frequency of Asn680Ser polymorphism of FSHR

\begin{tabular}{lccc}
\hline Genotype & Cases $(\mathbf{n = 1 0 0 )}$ & Controls $(\mathbf{n = 1 0 0 )}$ & P value \\
\hline Asn/Asn (AA) & $30(30 \%)$ & $32(32 \%)$ & \\
Asn/Ser (AS) & $42(42 \%)$ & $34(34 \%)$ & 0.689 \\
Ser/Ser (SS) & $28(28 \%)$ & $34(34 \%)$ & \\
\cline { 1 - 2 } Allele frequency & & & \\
\cline { 1 - 2 } Asn (A) & $98(49 \%)$ & $102(51 \%)$ & \multirow{2}{*}{0.777} \\
\hline Ser (S) & $102(51 \%)$ & $98(49 \%)$ & \\
\hline
\end{tabular}

We further analyzed some clinical and endocrine parameters for Asn680Ser polymorphism in the PCOS patients. There were no statistical differences in BMI, mFG score, levels of fasting glucose and fasting insulin, HOMA-IR, AND also levels of FSH, LH, E2, PRL, TSH and TT among the PCOS patients carrying different genotypes of Asn680Ser polymorphism (Table 3). 
Polymorphism of Follicle-Stimulating Hormone Receptor Gene in PCOS

Table 3. Relationship between Asn680Ser genotypes and some clinical characteristics in PCOS patients

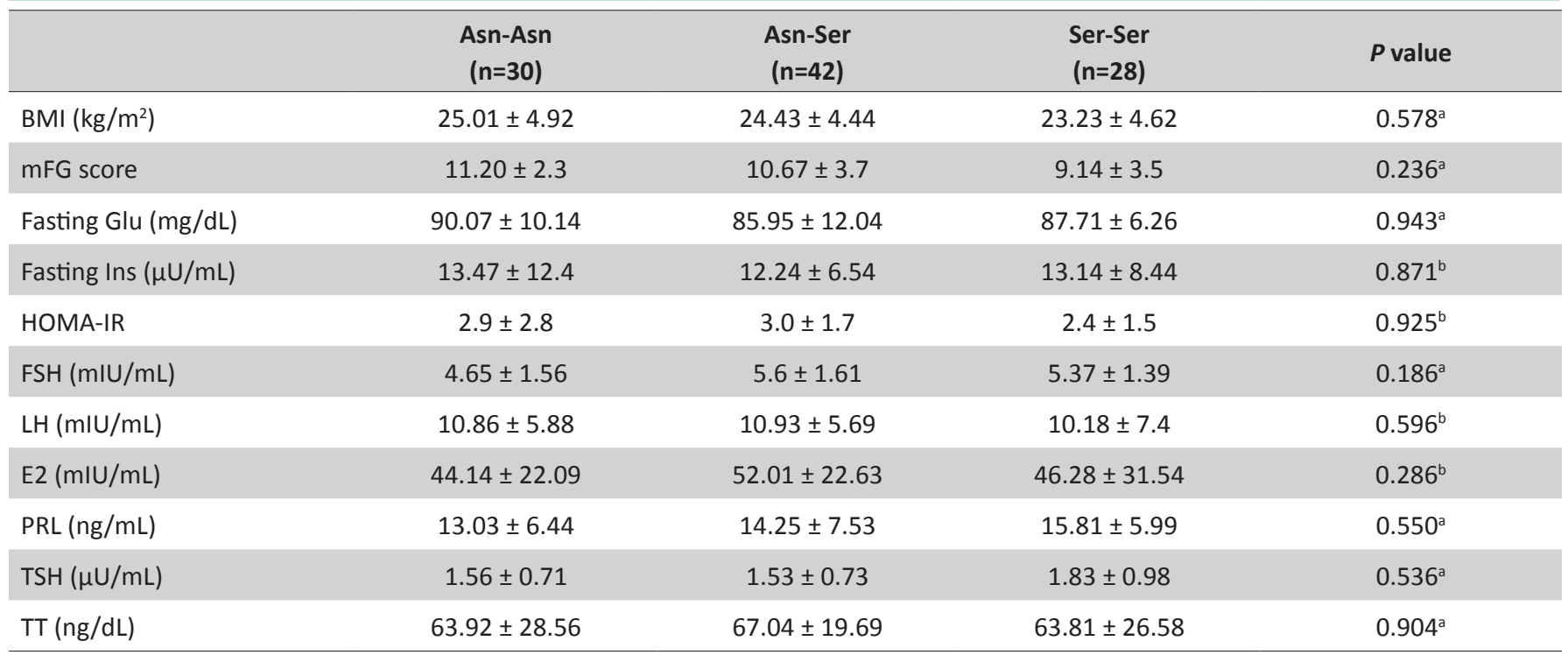

Data are presented as Mean \pm Standard deviation (SD)

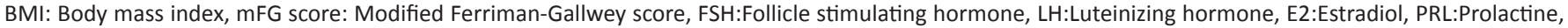
TSH:Thyroid stimulating hormone, TT:Total testosterone, HOMA-IR: Homoeostasis model assessment,

aNOVA

${ }^{\mathrm{b}}$ Kruskal Wallis test

\section{DISCUSSION}

Investigations on the distribution of Asn680Ser polymorphism of FSHR gene produced conflicting results, regarding ethnic differences. Balkan et al., evaluated the SNP of the FSHR at codon 680 in men from Southest Turkey and demonstrated that FSHR gene polymorphisms seemed not to have a direct effect on spermatogenesis, but were differently distributed, most likely due to the different genetic backgrounds in different populations [21]. The present study was first to report on the distribution of the SNP at codon 680 of the FSHR gene in Turkish women with PCOS from eastern provinces, comparing with the normal healthy controls. Our finding revealed that there were no differrences in both allelic frequency and genotypes for Asn680Ser polymorhism of FSHR between the PCOS patients and controls. Kuijper et al. reported the distribution of SNPs at codon 608 of the FSHR gene in a large population of infertile patients from various ethnic backgrounds. However, they found that a significantly higher number of Asian women have an Asn608 Asn variant, compared with Caucasians and Mediterraneans. In the same study, for Caucasian women, the distribution patterns were $29.6 \%$ for the Asn680Asn, $48.9 \%$ for the Asn680Ser, and 21.5\% for the Ser680Ser variant [22]. Similar results were reported by several researchers $[23,24]$. But, Laven et al. reported no difference in the genotype distribution of the FSHR (combination of the SNP) between fertility patient and controls in Dutch women. Nevertheless, they found a difference in the distribution of SNP at position 680 between anovulatory patients and controls [15]. Therefore, when comparing frequency distributions of FSHR polymorphic variants, ethnic background should be considered and patient should be distinguished as fertile or infertile.
FSH is essential for normal reproductive functions. Due to the important roles of FSH on follicular growth and steroidogenesis in females, mutations in the FSHR gene could affect reproductive ability. Based on this data, genetic variations in the FSHR gene have been extensively studied in association with ovulatory dysfunction, especially to clarify the etiopathogenesis of PCOS. The polymorhism at codon 680 was firstly reported by Aiitomaki in 1995 [25]. Since then, this SNP was investigated in patients with or without PCOS. However, the results are inconsistent. In a meta-analysis, it was suggested that there might be a significant association between Asn680Ser polymorphism and PCOS [26]. In accordance, $\mathrm{Gu}$ et al have reported that Asn680Ser may be associated with PCOS in Korean population [9]. Similarly, Sudo et al. reported a significantly higher prevelance of Asn680Ser genotypes in Japanese PCOS women than in those who had regular ovulation [27]. On the otherhand, while a study from the southeast China showed a significant association between PCOS and Asn608Ser polymorphism of FSHR in Han ethnic women [28], another study in the same population from North China revealed no association between PCOS and this SNP [29]. In addition, researchers did not find an association between FSHR gene polymorphism at codon 680 and PCOS in Thai women [30]. Also, no association was found between PCOS and Asn680Ser polymorphism in a Caucasian population from Netherlands [8]. This finding was supported by the study conducted on Turkish adolescant girls with PCOS [31]. According to our results, PCOS seems not to be associated with Asn680Ser polymorphism in women from eastern Turkey. Given the data from the literature presented, studies conducted in different ethnical populations and/or in different geographic regions may lead to discrepant results of the interrelationship between FSHR polymorphisms and PCOS. 
Patients with PCOS are susceptible to infertility, obesity and insulin resistance [32]. Based on this data, we investigated whether there was a correlation between FSHR polymorphism at codon 680 and BMI in PCOS patients with respect to the genotypes. We observed no significant differences in BMI among different genotypes of Asn680Ser polymorphism of FSHR in PCOS. Similarly, Wu et al and Singhasena et al have reported no correlation between BMI and FSHR polymorhism in PCOS patients $[29,30]$.

Most women with PCOS, both obese and lean, have a degree of insulin resistance. Recently, it has been reported that abnormal serine phosphorylation of insulin receptor (IR) may lead to a defect in insulin action and also, polymorphic alleles of insulin receptor substrate 1 and 2 (IRS- 1 and IRS-2) have a functional impact on the insulin-resistant component of PCOS [3]. To our knowledge, our study was the first to investigate the association among IR, Ser680Asn polymorhism of FSHR and PCOS in literature. Our findings have indicated no association between IR and this SNP in PCOS.

We also assessed a panel of PCOS-associated endocrine hormones with respect to the genotypes of Asn680Ser polymorphism in PCOS. Our data showed no significant differences for the levels of FSH, LH, E2, PRL, TSH, and TT among AA, AS and SS variants in PCOS patients. There are similiar studies in literature, supporting our results $[22,31]$. In contrast, some studies have suggested that women with Ser680Ser have a higher ovarian threshold for FSH, resulting in higher serum FSH concentrations $[23,33]$. Additionally, the Asn680Ser polymorhism of FSHR was reported to be related to the levels of FSH and PRL while other PCOS-associated endocrine hormones were not [29]. In another study by Fu et al, this SNP was found to be associated with high levels of FSH and low E2 levels [12].

In conclusion, our results indicate that there is no association between Asn680Ser polymorphism of FSHR and PCOS in Turkish women from eastern provinces of Turkey. In addition, this SNP seems not to be associated with BMI, IR and PCOS-associated endocrine hormones. Based on the evidence, ethnicity and geographical region may influence the polymorphism pattern. Therefore, further study on a large sample of Turkish population is needed to explore the role of FSHR polymorphisms in PCOS

Conflict of interest: All of the authors declared no conflicts of interest related to this study.

Financial Disclosure: No financial support was received.

\section{REFERENCES}

1. The Rotterdam ESHRE/ASRM-Sponsored PCOS Consensus Workshop Group. Revised 2003 consensus on diagnostic criteria and long-term health risks related to polycystic ovary syndrome. Fertil Steril. 2004;81:1925 .

2. Frank S. Polycystic ovary syndrome. N Engl J Med. 1995;333:853-61.

3. Jakubowski L. Genetic aspects of polycystic ovary syndrome. Endokrynol Pol. 2005;56:285-93.
4. Elghblawl E. Polycystic ovary syndrome and female reproduction. Br J Nurs. 2007;16: 1118-21.

5. Norman RJ, Dewailly D, Legro RS, Hickey TE. Polycystic ovary syndrome. Lancet. 2007;370:685-97.

6. Deligeoroglou E, Kouskouti C, Christopoulos P. The role of genes in the polycystic ovary syndrome: Predisposition and mechanisms. Gynecol Endocrinol. 2009;25: 603-9.

7. Simoni M, Tempfer CB, Destenaves B, Fauser BC. Functional genetic polymorphisms and female reproductive disoreders: Part I: Polycystic ovary syndrome and ovarian response. Hum Reprod Update. 2008;14:45984.

8. Valkenburg O, Uitterlinden AG, Piersman D, Hofman A, Themmen AP, de Jong FH, et al. Genetic polymorphism of $\mathrm{GnRH}$ and gonadotrophic hormone receptors affect the phenotype of polycystic ovary syndrome. Hum Reprod. 2009;24:2014-22.

9. $\mathrm{Gu} \mathrm{BH}$, Park JM, Back KH. Genetic variations of follicle stimulating hormone receptor are associated with polycystic ovary syndrome. Int J Mol Med. 2010;26:107-12.

10. Gromoll J,Ried T, Holtgreve-Grez H, Nieschlag E, Gudermann T. Localization of the human FSH receptor to 2p21 using a genomic probe comprising exon 10. J Mol Endocrinol.1994;12:265-71.

11. Simoni M, Gromoll J, Niesclag E. The follicle-stimulating hormone receptor: biochemistry, molecular biology, physiology, and pathophysiology. Endocr Rev. 1997;18: 739-73.

12. Fu L, Zhang Z, Zhang A, Xu J, Huang X, Zheng Q,et al. Association study between FSHR Ala307Thr and Ser680Asn variants and polycystic ovary syndrome (PCOS) in Northern Chinese Han women. J Assist Reprod Genet. 2013; 30: 717-21.

13. Altmäe S, Hovatta O, Stavreus-Evers A, Salumets A. Genetic predictors of controlled ovarian hyperstimulation: where do we stand today? Hum Reprod Update. 2011;17:813-28.

14. La Marca A, Sighinolfi G, Argento C, Grisendi V, Casarini L, Volpe A, Simoni M. Polymorphisms in gonadotropin and gonadotropin receptor genes as markers of ovarian reserve and response in in vitro fertilization. Fertil Steril. 2013; 99:970-8.

15. Laven JS, Mulders AG, Suryandari DA, Gromoll J, Nieschlag E, Fauser BC, Simoni M. Follicle-stimulating hormone receptor polymorphisms in women with normogonadotrophic anovulatory infertility. Fertil Steril. 2003;80: 986-992.

16. Mohiyiddeen L, Newman WG, McBurney H, Mulugeta B, Roberts SA, Nardo LG. Follicle-stimulating hormone receptor gene polymorphisms are not associated with ovarian reserve markers. Fertil Steril. 2012; 97:677-81.

17. Revised 2003 consensus on diagnostic criteria and long-term health risks related to polycystic ovary syndrome (PCOS). Hum Reprod. 2004;19: 263-65.

18. Katulski K, Slawek S, Czyzyk A, Podfigurna-Stopa A, Paczkowska K,. Ignaszak N, Podkowa N, Meczekalski B. Bone mineral density in women with polycystic ovary syndrome. J Endocrinol Invest. 2014; 37: 12191224.

19. Manco M, Castagneto-Gissey L, Arrighi E, Carnicelli A, Brufani C, Luciano R, Mingrone G. Insulin dynamics in young women with polycystic ovary syndrome and normal glucose tolerance across categories of body mass index. PLoS One. 2014; 9: e92995

20. Matthews D. R., Hosker J. P., Rudenski A. S., Naylor B. A., Treacher D. F., Turner R. C. Homeostasis model assessment: insulin resistance and $\beta$-cell function from fasting plasma glucose and insulin concentrations in man. Diabetologia. 1985;28:412-419.

21. Balkan M, Gedik A, Akkoc H, Izci Ay O, Erdal ME, Isi H, et al. FSHR single nucleotide polymorphism frequencies in proven fathers and infertile men in Southeast Turkey. J Biomed Biotechnol. 2010;2010:640318. 
22. Kuijper EA, Blankenstein MA, Luttikhof LJ, Roek SJ, Overbeek A, Hompes PG, Twisk JW, Lambalk CB. Frequency distribution of polymorphisms in the FSH receptor gene in infertility patients of different ethnicity. Reprod Biomed Online. 2010;20:588-93.

23. Perez Mayorga M, Gromoll J, Behre HM, Gassner C, Nieschlag E, Simoni M. Ovarian response to follicle-stimulating hormone (FSH) stimulation depends on the FSH receptor genotype. J Clin Endocrinol Metab. 2000;85:3365-9.

24. Behre HM, Greb RR, Mempel A, Sonntag B, Kiesel L, Kaltwasser P, Seliger E, Röpke F, Gromoll J, Nieschlag E, Simoni M. Significance of a common single nucleotide polymorphism in exon 10 of the folliclestimulating hormone $(\mathrm{FSH})$ receptor gene for the ovarian response to FSH: a pharmacogenetic approach to controlled ovarian hyperstimulation. Pharmacogenet Genomics. 2005;15:451-6.

25. Aittomäki K, Lucena JL, Pakarinen P, Sistonen P, Tapanainen J, Gromoll J, Kaskikari R, Sankila EM, Lehväslaiho H, Engel AR, Nieschlag E, Huhtaniemi I, de la Chapelle A. Mutation in the follicle-stimulating hormone receptor gene causes hereditary hypergonadotropic ovarian failure.Cell. 1995;82:959-68.

26. Qiu L, Liu J, Hei QM. Association between two polymorphisms of follicle stimulating hormone receptor gene and susceptibility to polycystic ovary syndrome: a meta-analysis. Chin Med Sci J. 2015;30:44-50.

27. Sudo S, Kudo M, Wada S, Sato O, Hsueh AJ, Fujimoto S. Geenetic and functional anayses of polymorhisms in the human FSH eceptor gene. Mol Hum Reprod 2002;8:893-99.
28. Du J, Zhang W, Guo L, Zhang Z, Shi H, Wang J, Zhang H, Gao L, Feng G, He L.Two FSHR variants, haplotypes and meta-analysis in Chinese women with premature ovarian failure and polycystic ovary syndrome. Mol Genet Metab. 2010;100: 292-5.

29. Wu XQ, Xu SM, Liu JF, Bi XY, Wu YX, Liu J. Association between FSHR polymorphisms and polycystic ovary syndrome among Chinese women in north China. J Assist Reprod Genet. 2014;31: 371-7.

30. Singhasena W, Pantasri T, Piromlertamorn W, Samchimchom S, Vutyavanich T. Follicle-stimulating hormone receptor gene polymorphism in chronic anovulatory women, with or without polycystic ovary syndrome: a cross-sectional study. See comment in PubMed Commons belowReprod Biol Endocrinol. 2014;12 86-92.

31. Unsal T, Konac E, Yesilkaya E, Yilmaz A, Bideci A, Ilke Onen H, Cinaz P, Menevse A. Genetic polymorphisms of FSHR, CYP17, CYP1A1, CAPN10, INSR, SERPINE1 genes in adolescent girls with polycystic ovary syndrome. J Assist Reprod Genet. 2009;26:205-16.

32. Norman RJ, Dewailly D, Legro RS, Hickey TE. Polycystic ovary syndrome. Lancet. 2007;370:685-97.

33. de Koning CH, Benjamins T, Harms P, et al. The distribution of FSH receptor isoforms is related to basal FSH levels in subfertile women with normal menstrual cycles. Hum Reprod. 2006;21:443-6. 